\title{
Orientational order parameter of liquid crystalline mixtures - A birefringence study
}

\author{
Shahina $^{1}$, C. M. Subhan ${ }^{2}$, S. Rangappa ${ }^{3}$, K. Fakruddin ${ }^{1 *}$ \\ ${ }^{1}$ Department of Physics, Ghousia College of Engineering, Ramanagara., Karnataka, India \\ ${ }^{2}$ Department of Physics, Shri Shirdi Sai Institute of Science and Engineering, Vadiyampeta, \\ Anantapurmu, Andhra Pradesh, India \\ ${ }^{3}$ Department of Physics, BIT, Bangalore, Karnataka, India \\ *drfakruddin2008@gmail.com
}

PACS 42.70.Df, 61.30.Gd,42.81.Gs,78.20.Ci

DOI 10.17586/2220-8054-2018-9-6-735-740

\begin{abstract}
The orientational order parameter is one of the most important material parameters of nematic phase. In the present work, the phase transition temperatures of the mesogenic mixtures are measured by using a polarizing optical microscope. The birefringence in nematic phase is estimated by measuring ordinary and extraordinary refractive indices. The birefringence is also measured by measuring the diameter of Newton's rings at various temperatures in nematic phase. The orientational order parameter is evaluated by using the birefringence data obtained in the above two methods. The orientation order parameter can be enhanced by the dispersion of different nanoparticles in the liquid crystal matrix.
\end{abstract}

Keywords: Liquid crystalline mesogens, orientational order parameter, birefringence, refractive index, Newtons rings.

Received: 17 August 2018

Revised: 27 October 2018

\section{Introduction}

Liquid crystals are self-assembled dynamic functional soft materials which posses both order and mobility at molecular, supramolecular and microscopic levels [1,2]. Liquid crystals have received much attention in the recent years because they exhibit anisotropy in their mechanical, electrical and optical properties, behaving like solid crystals nevertheless they have no ability to support shearing and thus they flow like ordinary isotropic solution. This dual nature of the materials is important in technological applications such as displays and optical switches. With technological advances the LC display (LCD) is currently expected to achieve high electro optical performance as well as to be of low power consumption [3]. Instead of synthesizing a number of LC compounds as constituents, either a suitable mixtures can be used or by doping nano materials into LCs can be used. By mixing two nematogens, one can often obtain a lower melting point. Usually two nematogens exhibit the property of continuous miscibility without crossing any line [4]. Binary mixtures offer the advantage of frequently exhibiting eutectic behavior in their solid mesophase transition and in mesophase isotropic transition. The temperature varies linearly with composition further for the technological use of LC; the liquid crystalline materials of specific molecular design and synthesis are viable but expensive to influence the properties of mesophases. The desired properties can be obtained by mixing compounds of various molecular shapes rather than by looking for pure compounds $[5,6]$ or by the incorporation of metal nanoparticles into LCs, which makes it easier to obtain a better profile of display parameters [7]. The metal nanoparicles embedded in LCs have attracted much interest not only in the field of magnetic recording media but also in the area of medical care including hyperthermia, photomagnetics, magnetic resonance imaging, cancer therapy, sensors and high frequency applications [8-12].

The amino methyl hydroxyl phenoxycarbonitrile and amino phenyl benzoates are the exciting class of new LC compounds with different spacer, end and terminal groups having fascinating structural variations. In the present work, the following homologous series of compounds are selected and their mixtures are taken in equal ratio. The molecular structure of the liquid crystal compounds in the present work is shown in Fig. 1. The optical studies were carried out; using the data obtained and the orientational order parameter by birefringence methods is evaluated.

\section{Experimental}

\subsection{Refractive index method to measure birefringence}

The refractive indices of the liquid crystalline compounds were measured with wedge shaped glass cell similar to the one used to obtain birefringence by Haller et al. $[13,14]$ and modified spectrometer. A wedge shaped glass cell was prepared with two optical flats of rectangular glass plates $(50 \mathrm{~mm} \times 25 \mathrm{~mm})$ sandwiched with glass slide of $0.05 \mathrm{~mm}$ thick which acted as a wedge spacer. The cell is filled with the liquid crystal material. The liquid 

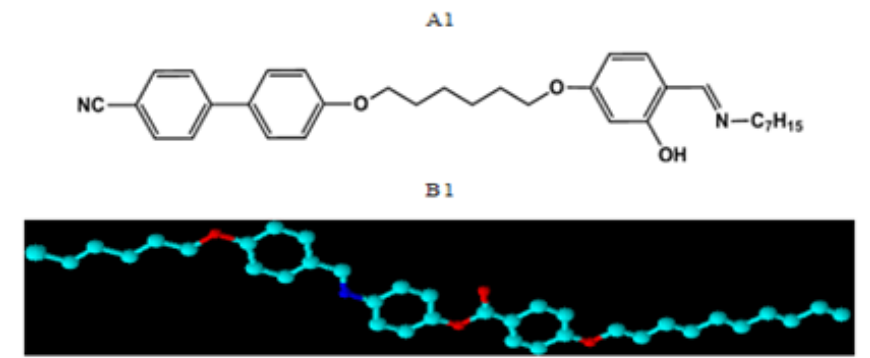

A.2

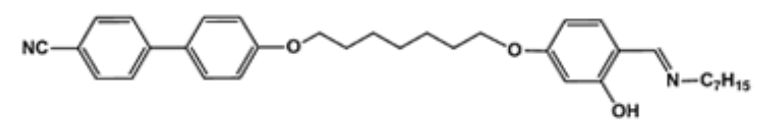

$\mathrm{B} 2$

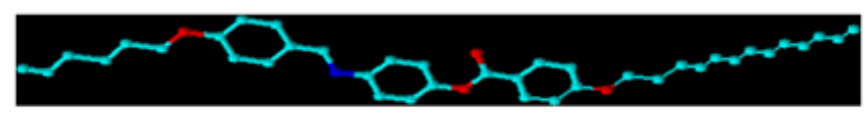

FIG. 1. Molecular structure of the $\mathrm{LC}$ compounds $\mathrm{C} 1=\mathrm{A} 1+\mathrm{B} 1 \& \mathrm{C} 2=\mathrm{A} 2+\mathrm{B} 2$

A1: 41-(6-(4-(heptylamino)methyl)-3-hydroxyphenoxy)hexyloxy)biphenyl-4-carbonitrile $\left[\mathrm{C}_{33} \mathrm{H}_{40} \mathrm{~N}_{2} \mathrm{O}_{3}\right]$

B1: 4-((4-(hexyloxy)benzylidene)amino)phenyl4-(decycloxy)benzoate $\left[\mathrm{C}_{36} \mathrm{H}_{47} \mathrm{~N}_{1} \mathrm{O}_{4}\right]$

A2: 41-(7-(4-(pentylamino)methyl)-3-hydroxyphenoxy)heptyloxy)biphenyl-4-carbonitrile $\left[\mathrm{C}_{34} \mathrm{H}_{42} \mathrm{~N}_{2} \mathrm{O}_{3}\right]$

B2: 4-((4-(hexyloxy)benzylidene)amino)phenyl4-(tridecycloxy)benzoate $\left[\mathrm{C}_{39} \mathrm{H}_{53} \mathrm{~N}_{1} \mathrm{O}_{4}\right]$

crystal material in the cell acts as a uniaxial crystal with its optic axis parallel to the edge of the spacer glass plate. The same technique is also used by several authors [15-17].

\subsection{Newton's ring method to measure birefringence}

The experimental setup consists of Plano convex lens of small radius of curvature $(13 \mathrm{~mm})$ and plane glass plate which is being placed in hot stage connected to specially designed microcontroller based temperature and image capturing device. The LC sample whose birefringence is to be measured is placed between the lens and plate. Systems of concentric rings were observed in nematic phase. The optical path difference between e-ray and o-ray is given by $y, \delta n$ which corresponds to ring number $k$ and wavelength $\lambda$ for a bright fringe is given by:

$$
\begin{gathered}
\delta n=\frac{k \lambda}{y}, \\
y=\frac{x^{2}}{2 R} .
\end{gathered}
$$

From Eqns. (1) and (2),

$$
\delta n=\frac{(2 R \lambda) k}{x^{2}}
$$

Since $2 R \lambda=C$, cell constant for the given wavelength of light:

$$
\delta n=\frac{C k}{x^{2}}
$$

where $x$ is the radius of the ring and $R$ is the radius of curvature of the lens used. Thus, $\delta n$ can be measured with great accuracy by taking the slope of the straight line drawn between $x^{2}$ versus the ring number, $k$. We can obtain the same result by considering the dark rings also; as the temperature decreases, birefringence $\delta n$ increases. The method adopted for the estimation of orientational order parameter from $\delta n$ given by Kuczynski et al. [18, 19].

\subsection{Estimation of order parameter from birefringence $(\delta n)$}

For the determination of order parameter $S$ from the birefringence measurements $\delta n$ without considering the local field experienced by the molecule in a LC phase were used by many authors [20-22]. The birefringence $\delta n$, which is a function of temperature, is fitted to the following equation:

$$
\delta n=\Delta n\left(1-\frac{T}{T^{*}}\right)^{\beta}
$$


TABLE 1. The phase variants and transition temperatures and thermal ranges of mesogenic mixtures

\begin{tabular}{|c|c|c|c|c|c|c|c|}
\hline \multirow{2}{*}{$\begin{array}{c}\text { S1. } \\
\text { No. }\end{array}$} & Compound & \multicolumn{4}{|c|}{ Transition Temperature ${ }^{\circ} \mathrm{C}$} & \multicolumn{2}{c|}{ Thermal range } \\
\cline { 3 - 8 } & & $\mathrm{I}-\mathrm{N}$ & $\mathrm{N}-\mathrm{SmA}$ & $\mathrm{SmA}-\mathrm{SmB}$ & Crystal & $\Delta \mathrm{N}$ & $\Delta \mathrm{SmA}$ \\
\hline 1 & $\mathrm{C} 1=50 \% \mathrm{~A} 1+50 \% \mathrm{~B} 1$ & 184.6 & 169 & 160 & 85.6 & 15.6 & 9 \\
\hline 2 & $\mathrm{C} 2=50 \% \mathrm{~A} 2+50 \% \mathrm{~B} 2$ & 137.8 & 126.4 & 109 & 67.1 & 11.4 & 17.4 \\
\hline
\end{tabular}

where $T$ the absolute temperature, $T^{*}$ and $\beta$ are constants, $T^{*}=T+X$, where $X$ is varied from 0.0001 to $4 \mathrm{~K}$ and the exponent $\beta$ is close to $0.17-0.22$. This procedure enables one to extrapolate $\delta n$ to the absolute zero temperature. In practice, the three adjustable parameters $T, \Delta n$ and $\beta$ were obtained by fitting the experimental data for $\delta n$ to the following equation written in the logarithmic form:

$$
\log \delta n=\log \Delta n+\beta \log \left(\frac{T^{*}-T}{T^{*}}\right) .
$$

In these investigations, the values $\log \Delta n$ and $\beta$ are estimated by the linear regression method. The parameter $T^{*}$ is adjusted to get best correlation coefficient of the linear regression $\left(T^{*}\right.$ is higher than the clearing temperature and is adjusted up to $4 \mathrm{~K}$ to obtain the best correlation regression coefficient of linear regression). Further, in evaluating $\Delta n$, only the nematic range is considered. Thus, $S$ is given by:

$$
S=\frac{\delta n}{\Delta n}
$$

\section{Results and discussion}

The phase variants and transition temperatures of the above mesogenic mixtures are measured by using polarizing thermal microscope and depicted in Table 1. The refractive indices of the mesogenic mixtures are measured at various temperatures by using modified spectrometer attached with small angled prism which houses the LC sample. Anisotropy is an inherent property of liquid crystals therefore it exhibits birefringence. When the temperature dependence of refractive index (R.I) is measured, the R.I remains constant in isotropic phase. With the decrease of temperature from I-N phase, the isotropic R.I value splits into two, extraordinary R.I $\left(n_{e}>n_{i s o}\right)$ and ordinary R.I $\left(n_{o}<n_{i s o}\right)$ respectively. This splitting is clearly observed in the telescope of spectrometer at an angle of minimum deviation. When the temperature decreases, ne increases while the no decreases. With the further decrease of temperature in the deep nematic region the R.I value of both ne and no attains deep saturation which is shown in Fig. 2. It is also observed that the refractive indices could not be measured in smectic phases as the splitting has hazy appearance.
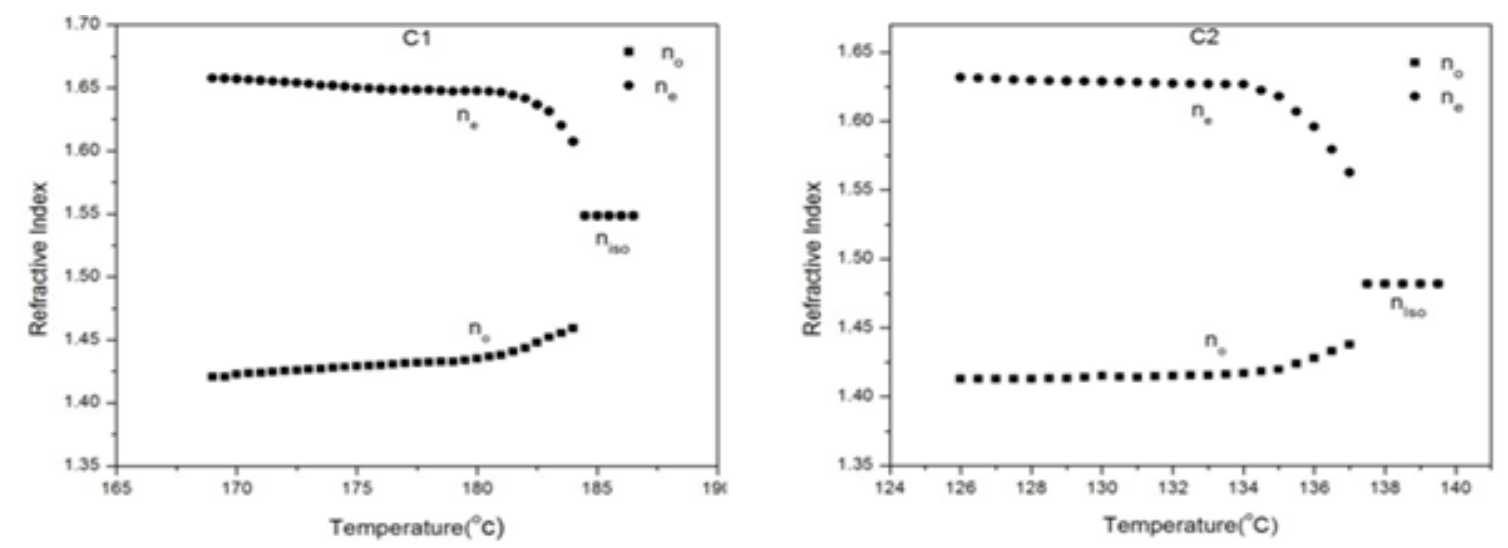

FIG. 2. The temperature variation of Refractive index in $\mathrm{C} 1 \& \mathrm{C} 2$ mixtures

While evaluating the $S$ by refractive indices and Newton's rings methods the parameter $T^{*}$ is adjusted to $T_{C}+(1.5-2.2 \mathrm{~K})$. The birefringence in perfect order and $\beta$ values obtained are represented in Table 2 . 
TABLE 2. The birefringence of compounds in perfect order and $\beta$ values

\begin{tabular}{|c|c|c|c|c|c|}
\hline \multirow{2}{*}{$\begin{array}{c}\text { Sl. } \\
\text { No. }\end{array}$} & \multirow{2}{*}{ Compound } & \multicolumn{2}{|c|}{ Birefringence in perfect order } & \multicolumn{2}{|c|}{$\beta$ (values) } \\
\cline { 3 - 6 } & & RI method & Newton rings method & RI method & Newton rings method \\
\hline 1 & C1 & 0.42 & 0.420 & 0.19 & 0.189 \\
\hline 2 & C2 & 0.40 & 0.417 & 0.22 & 0.200 \\
\hline
\end{tabular}

In the liquid crystalline phase both Brownian and crystalline fields are acting, which results the decrease of potential on the electron system,thus contributing to more polarization. Hence the birefringence increases with decrease of temperature. As $\log \left(1-T / T^{*}\right)$ decreases $\log (\delta n)$ increases as shown in Fig. 3.
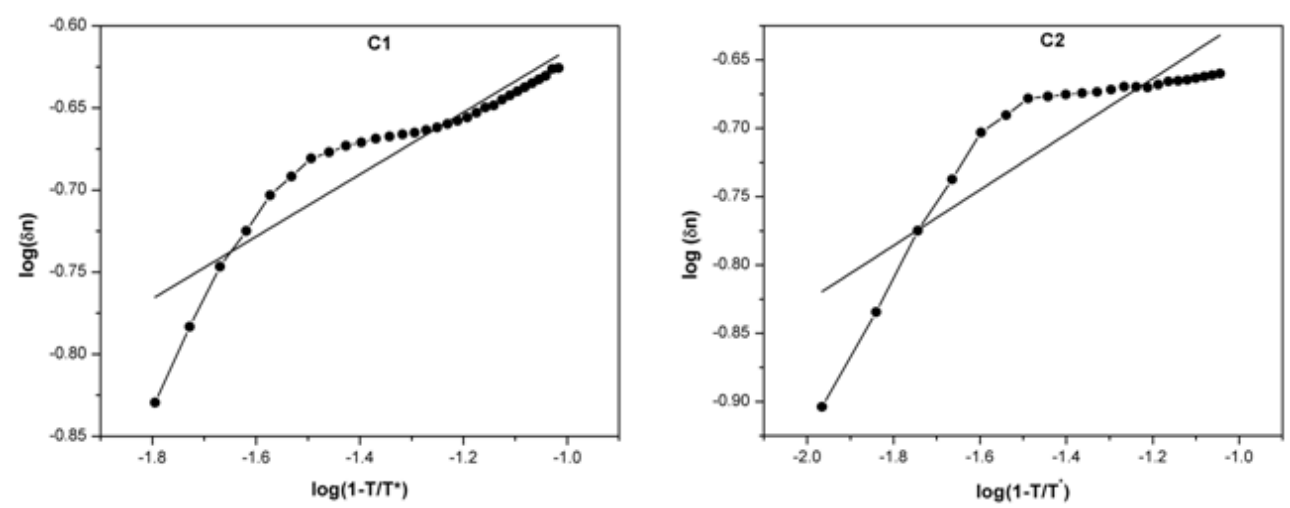

FIG. 3. The $\log (\delta n)$ vs $\log \left(1-T / T^{*}\right)$ by RI method for $\mathrm{C} 1 \& \mathrm{C} 2$

The diameter of Newton's rings is directly proportional to square root of natural numbers. The birefringence $\delta n$ is measured by Newton's rings method by measuring the radius of the Newton's rings at various temperatures. The slope of the straight line is estimated by plotting the graph between the square of the radius of the ring vs. the ring number at $518 \mathrm{~nm}$ for I-N temperatures. It is observed that as the temperature increases the slope increases and birefringence decreases. This variation is represented in the Fig. 4 for $\mathrm{C} 1$ and $\mathrm{C} 2$ mixtures.
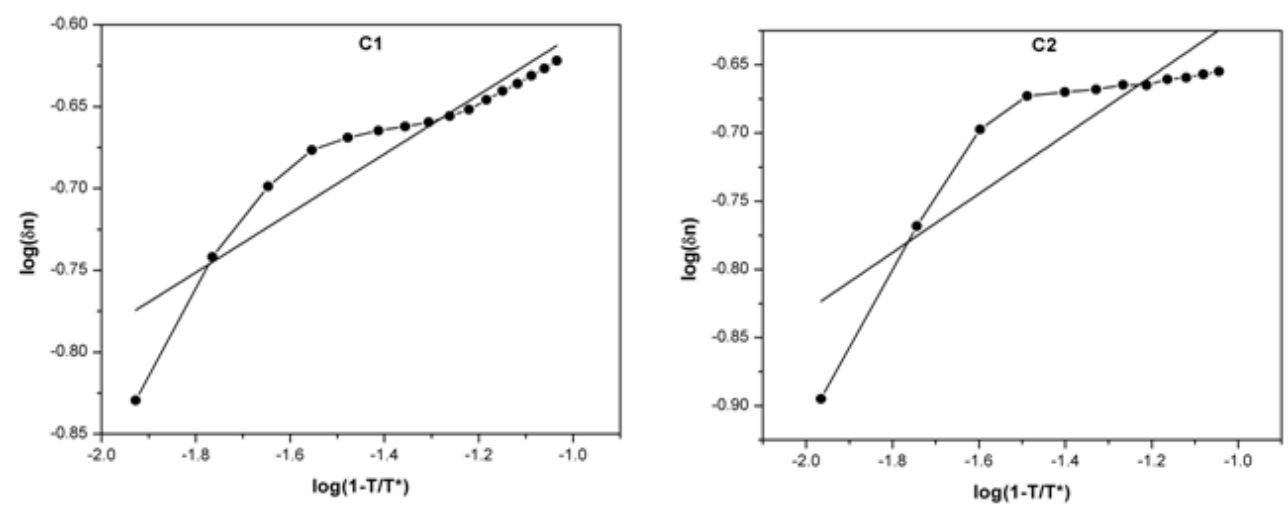

FIG. 4. The $\log (\delta n)$ vs $\log \left(1-T / T^{*}\right)$ in newton rings method of $\mathrm{C} 1 \& \mathrm{C} 2$

As the LC transitions takes place from I-N, the packing of the molecules occurs with positional as well as translational order, hence order parameter $(S)$ increases with decrease of temperature. The OOP obtained by birefringence methods are in the range of $0.3-0.7$ for the above LC mixtures. The $S$ values obtained from $\delta n$ by refractive index and Newton rings method are in good agreement with each other. The comparison of the $S$ by the two optical methods is shown in the Fig. 6. 

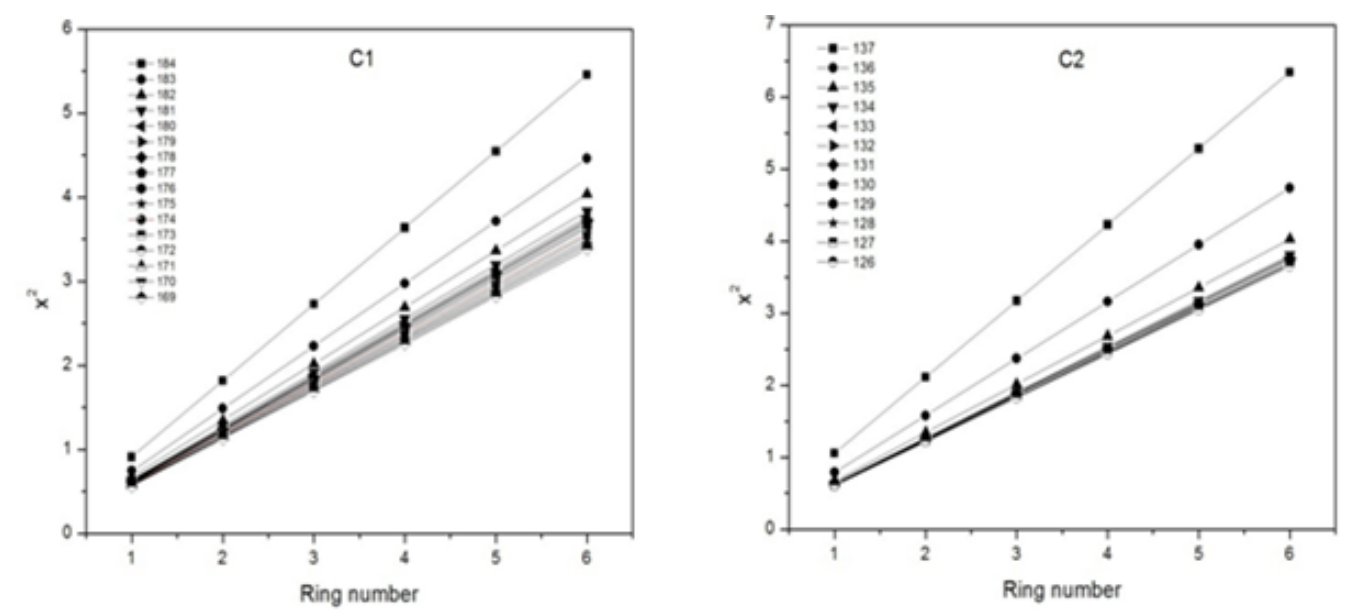

FIG. 5. The variation of ring number and square of radius in $\mathrm{C} 1 \& \mathrm{C} 2$ compounds
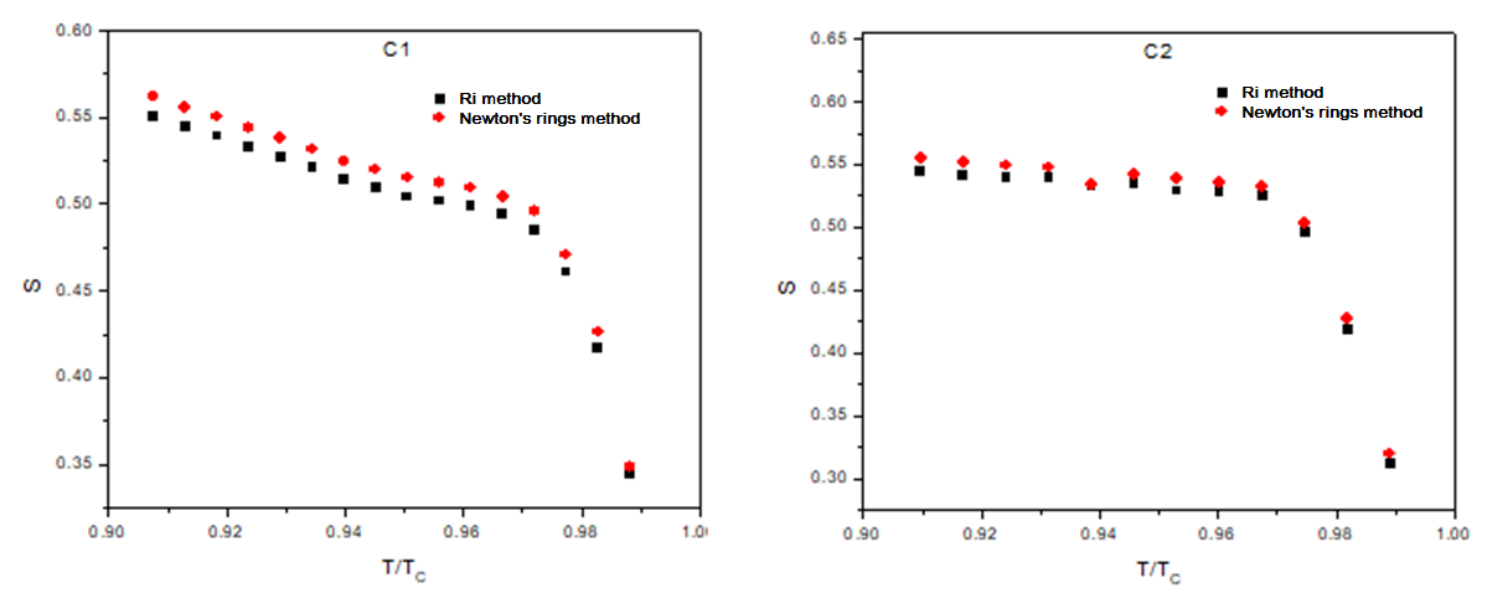

FIG. 6. The variation of $S$ with reduced temperature in $\mathrm{C} 1 \& \mathrm{C} 2$ mixtures

\section{Summary}

The use of Newton's rings technique is a precise method for the determination of birefringence of different liquid crystalline phases using simple approximations. This method can also be applied to study the order parameter in smectic phases, as no local field is involved. The order parameter obtained by Newton's rings method is compared with that of refractive indices method. The liquid crystals, being anisotropic media, hence provide good support for the self assembly of nano materials into large organizing structures in multiple dimensions. Therefore, dispersion of nano particles into liquid crystals has emerged as fascinating area of research from an application point of view. Nano objects (guests) that are embedded in the liquid crystals (hosts) can trap the ion concentration, electrical conductivity and improve electro optic response of the host materials. Hence, by the dispersion of nanoparticles into liquid crystals, one can improve the orientation order parameter, an important display property.

\section{Acknowledgements}

Authors express their thanks to the Department of Physics, Ghousia College of Engineering, Ramanagaram for providing laboratory facilities. The authors are thankful to Dr. C. M. Yellamagad and Dr. D. S. ShankarRao for providing liquid crystalline compounds to carry out the present studies. The financial support rendered by the VTU Belgaum under VTU research grant scheme for the research project [No. VTU/Aca/2011-12/A-9/750] is gratefully acknowledged. 


\section{References}

[1] Kato T., Mizoshita N., Kishimoto K. Functional liquid-crystalline assemblies: self-organized soft materials. Angew Chem. Int. Ed. Engl., 2005, 45 (1), P. 38-68.

[2] Goodby J.W., Saez I.M., et al. Transmission and amplification of information and properties in nanostructured liquid crystal. Angew Chem. Int. Ed. Engl., 2008, 47 (15), P. 2754-2787.

[3] Shiraishi Y., Toshima N., et al. Frequency modulation response of a liquid-crystal electro-optic device doped with nanoparticles. Appl. Phys. Lett., 2002, 81 (15), P. 2845-2847.

[4] de Gennes P.G., Prost J. The Physics of Liquid Crystals, Clarendon Press, Oxford, 1998.

[5] Obadovic D.Z., Stojanovic M., et al. Study of Binary Cholesteric Liquid Crystalline Mixtures Doped with Some Chiral Nonmesogenic Estradiol Derivatives. Mol. Cryst. Liq. Cryst., 2011, 547, P. 1736-1743.

[6] Eren San S., Mustafa Okutan, Oguz Koysal, Yusuf Yerli. Carbon Nanoparticles in Nematic Liquid Crystals. Chin. Phys. Lett., 2008, 25 (1), P. 212-215.

[7] Goel P., Arora M., Biradar A.M. Electro-optic switching in iron oxide nanoparticle embedded paramagnetic chiral liquid crystal via magneto-electric coupling. J. App. Phy., 2014, 115, P. 124905.

[8] Silva J.B., Brito W.D., Mohallem N.D.S. Influence of heat treatment on cobalt ferrite ceramic powders. Material Sci. Eng. B, 2004, 112, P. $182-187$.

[9] Sun S., Murray C.B., et al. Monodisperse FePt Nanoparticles and Ferromagnetic FePt Nano Crystal Superlattices. Science, 2000, 287, P. 1989-1992.

[10] Pankhurst Q.A., Connolly J., Jones S.K., Dobson J. Applications of magnetic nanoparticles in biomedicine. J. Phys. D: Appl. Phys., 2003, 36, P. 167-181.

[11] Ito A., Shinkai M., Honda H., Kobayashi T. Medical application of functionalized magnetic nanoparticles. J. Bio. Sci Bio. Eng., 2005, 100 (1), P. 1-11.

[12] Meng X., Li H., et al. Mossbauer study of cobalt ferrite nanocrystals substituted with rare-earth $\mathrm{Y}^{3+}$ ions. J. Magn. Magn. Meter., 2009, 321, P. 1155-1158.

[13] Toh C.L., Xu J., He C. Synthesis and characterisation of main-chain hydrogen-bonded supramolecular liquid crystalline complexes formed by azo-containing compounds. Liq. Cryst., 2008, 35 (3), P. 241-251.

[14] Haller I., Huggins H.A., Lilienthal H.R., McGuire T.R. Order - Related Properties of Some Nematic Liquids. J. Phys. Chem., 1973, 77 (7), P. 950-954.

[15] Fakruddin K., JeevanKumar R., DattaPrasad P.V., Pisipati V.G.K.M. Orientational Order Parameter - 1 A Birefringence Study. Mol. Cryst. Liq. Cryst., 2009, 511, P. 133-145.

[16] Subhan C.M., Jeevan Kumar R., et al. An Optical Study to Estimate Orientational Order Parameter on Some Benzylidene amino phenyl benzoate Liquid Crystals. Acta Physica Polonica A, 2016, 129 (3), P. 284-288.

[17] Narasimhamurthy G.K., Subhan C.M., et al. Orientational order parameter of some CBOnO.m liquid crystalline compounds - an optical study. Mol. Cryst. Liq. Cryst., 2016, 641, P. 25-36.

[18] Kuczynski W., Zywucki B., Malecki J. Determination of Orientational Order Parameter in Various Liquid-Crystalline Phases. Mol. Cryst. Liq. Cryst., 2002, 381, P. 1-19.

[19] Zywucki B.J., Kuczynski W. The Orientational Order in Nematic Liquid Crystals From Birefringence Measurements. Dielectr. Electr. Insul., 2001, 8 (3), P. 512-515.

[20] Ramakrishna Nanchara Rao M., Datta Prasad P. V., Pisipati V.G.K.M., MadhaviLatha D. An Optical study on Orientational order parameter and Molecular Polarizability in Benzylidene Anilines. Solid State Phenomena, 2012, 181-182, P. 102-105.

[21] Subramhanyam H.S., Krishnamurti D. Polarization Field and Molecular Order in Nematic Liquid Crystals. Mol. Cryst. Liq. Cryst., 1973, 22, P. 239-248.

[22] Pardhasaradhi P., Datta Prasad P.V., et al. Orientational order parameter studies in two symmetric dimeric liquid crystals - an optical study. Phase Transitions, 2012, 85 (12), P. 1031-1044. 